\title{
The role of discontinuities in the perception of subjective figures
}

\author{
THOMAS F. SHIPLEY and PHILIP J. KELLMAN \\ Swarthmore College, Swarthmore, Pennsylvania
}

\begin{abstract}
Recently we proposed a theory of visual interpolation (Kellman \& Shipley, in press) that addresses a variety of unit formation phenomena, including the perception of partly occluded objects and subjective figures. A basic notion of the theory is that discontinuities in the first derivative of projected edges are the initiating conditions for interpolation of boundaries that are not physically specified. In this paper, we report four experiments in which this claim was tested in the domain of subjective figures. Experiments 1 and 2 demonstrate that discontinuities in the first derivative of the edges of inducing elements have a clear effect on the frequency of report and the perceived clarity of simple subjective figures. Similar effects are found when unfamiliar subjective figures and inducing elements are used (Experiment 3). Experiment 4 rules out the possibility that the discontinuities in the first derivative merely add to the clarity of subjective figures. These experiments suggest that first-order discontinuities play a central role in unit formation.
\end{abstract}

All subjective figure displays from the earliest (Schumann, 1904) to the most recent share a common attribute. This attribute is not to be found on any list of the factors that have most often been proposed to explain subjective contours. Such a list would contain at least the following: figural symmetry (van Tuijl \& Leeuwenberg, 1982), familiarity (Rock, 1983), collinearity of edges, brightness differences between adjacent areas (Brigner \& Gallagher, 1974), implicit interposition cues (Coren, 1972; Parks, 1986; see also Day, 1987), figures containing concave gaps (Kanizsa, 1979), and perceptual set (Wallach \& Slaughter, 1988). Yet, for every one of these factors, robust subjective figures have been created in which that factor is absent. (For a review, see Kellman \& Shipley, in press.) This fact has led some to theorize in terms of multiple determinants of subjective contours and figures (e.g., Day, 1987; Halpern, 1981).

There is, however, a feature that characterizes every clear case of subjective figure perception. A general theory of subjective figures, as well as other unit formation phenomena, may be feasible with this feature as its central concept. Recently, we have proposed just such a theory (Kellman \& Loukides, 1987; Kellman \& Shipley, in press). The critical feature common to all subjective figure displays is the presence of discontinuities in the first

This research was supported by NSF Research Grant BNS 85-19851 and a Swarthmore College Faculty Research Grant to P.J.K. Portions of this research were presented at the 29th Annual Meeting of the Psychonomic Society (1988). We wish to thank Dave Dickter and Corrie Philips for help in collecting some of the data, and the members of T. F. Shipley's thesis committee-Henry Gleitman, Ed Pugh, and Mike Kelly-for their guidance. Reprint requests should be addressed to Thomas F. Shipley, Department of Psychology, Swarthmore College, Swarthmore, PA 19081. derivative of the bounding contours of the inducing patterns. In other words, all good subjective figure displays appear to have inducing elements with abrupt changes in the slope of their outer boundaries. Hereafter, we will use the symbol $D_{1}$ to refer to such a discontinuity in the first derivative. In Figure 1, the sharp corners on the black figures are $D_{1} s . D_{1} s$ in the optical projection invariably occur in cases of occlusion (see Kellman \& Shipley, in press), as well as when objects with sharp corners are projected. ${ }^{1}$ The ecological facts suggesting the usefulness of $D_{1} s$ in object perception, and the additional considerations required in their use, are discussed elsewhere (Kellman \& Loukides, 1987; Kellman \& Shipley, in

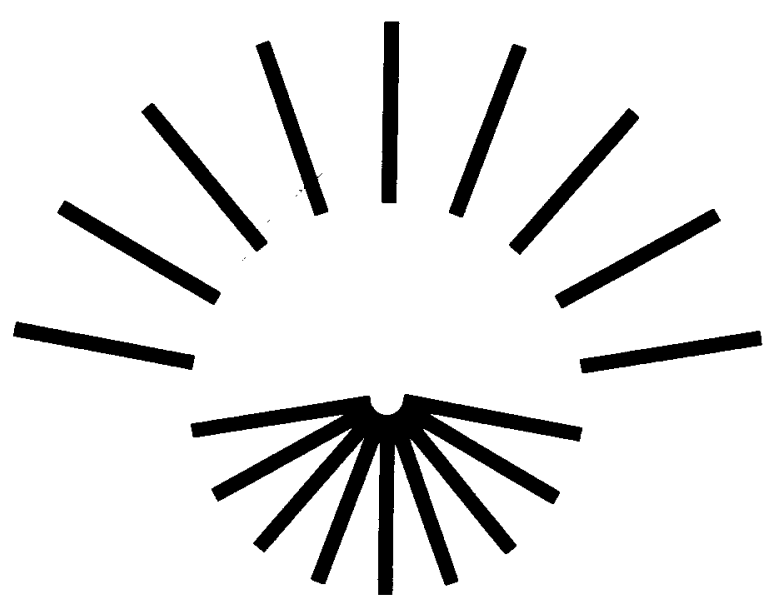

Figure 1. An example of a subjective figure. Eighteen black rectangles serve as the inducing elements. There is a discontinuity in the first derivative of the boundary of each rectangle at each of its four corners. 
press). In this paper, we will focus on the role of discontinuities in subjective figure perception.

The discontinuity theory holds that $D_{1} s$ in the inducing elements' boundaries are necessary for the perception of subjective figures. Interpolation of contours should not occur unless $D_{1} s$ are present in the display. A survey of the literature (e.g., Petry \& Meyer, 1987) suggests that all displays that produce clear subjective figures contain $D_{1} s$. Furthermore, both Sambin (1987) and Kennedy (1978) have observed that rounding off the ends of thin rectangular inducing elements results in the loss of perceived subjective edges. Rounding off the ends of such rectangles removes the $D_{1} s$ in the boundaries of the inducing elements. The lack of subjective figure displays that do not contain $D_{1} s$ in the inducing elements, along with the observations made by Sambin and Kennedy, suggests that $D_{1} s$ are important for the perception of subjective figures. However, there has been no explicit or systematic test of this possibility. In the present experiments, we tested the necessity of $D_{1} s$ for the perception of subjective figures.

\section{EXPERIMENT 1}

Subjective figure displays were constructed containing clear $D_{1} s$ at the points where subjective edges begin and end. A second set of displays was constructed, similar to these in all respects except that the inducing elements were rounded off where the $D_{1} s$ had been. Specifically, the areas of the inducing elements, the shape of the potential subjective figure, and the amount of inducing element bordering the subjective figure were the same as in the displays with $D_{1} s$. Subjects were asked to report the presence or absence of a subjective figure, and a magnitude estimation procedure was used to assess contour clarity.

\section{Method}

Subjects. Twenty-four undergraduates at the University of Pennsylvania served as subjects in 30-min individual testing sessions. Each subject received $\$ 2.50$ for participating. None of the subjects had taken a perception class.

Apparatus. All stimuli were designed and presented on a Commodore Amiga computer with a Commodore 1080 RGB monitor that measured $20 \times 25 \mathrm{~cm}$. Using the computer's high resolution mode, the screen's resolution was $400 \times 640$ pixels. All stimuli were presented to each subject in a random order (except where noted), with the constraint that for any given subject who received a particular random order there was another subject who was presented with the stimuli in the reverse order.

The subjects were positioned $150 \mathrm{~cm}$ from the monitor. The only source of light in the room, other than the monitor, was a screened $100-\mathrm{W}$ bulb positioned above and behind the monitor. This lighting arrangement reduced reflections from the monitor.

Stimuli. Four pairs of figures were used. One member of each pair was a subjective figure display whose inducing elements contained sharp changes in the direction of the contour $\left(D_{1} s\right.$ present, DP). The other member of each pair was a display similar to the DP display, except that sharp changes in the direction of the inducing elements' contour were removed $\left(D_{1} s\right.$ absent, $\left.D A\right)$. The subjective figures possible in the four pairs were a triangle, a square, a rounded triangle (the corners of the triangle had been rounded off), and a circle. The dimensions of the figures used were: triangle, base $=7.7 \mathrm{~cm}\left(2.9^{\circ}\right.$ visual angle $)$, height $=6.6 \mathrm{~cm}\left(2.5^{\circ}\right.$ visual angle); square, base $=7.7 \mathrm{~cm}\left(2.9^{\circ}\right.$ visual angle); rounded triangle, base $=3.7 \mathrm{~cm}\left(1.4^{\circ}\right.$ visual angle $)$, height $=3.7 \mathrm{~cm}\left(1.4^{\circ}\right.$ visual angle); circle, radius $=4 \mathrm{~cm}\left(1.5^{\circ}\right.$ visual angle). Examples of two DA displays are shown on the left in Figures 2 and 3. The corresponding DP displays are shown on the right in Figures 2 and 3 . Three pairs of displays (triangle, square, and rounded triangle) were constructed by adding white circles equivalent in size to the inducing elements used to make the base display. ${ }^{2} \mathrm{~A}$ base display is shown in the center of Figure 2. The inducing elements used in the base displays had the following dimensions: triangle (Figure 2) and square, radius $=1.92 \mathrm{~cm}\left(0.7^{\circ}\right.$ visual angle); rounded triangle, radius $=1 \mathrm{~cm}\left(0.4^{\circ}\right.$ visual angle $)$. The circles were added to the base display in two different ways to form the stimulus pairs. For the DA displays, the circles were placed tangent to the contour of the inducing elements that specified an edge of the subjective figure, thereby producing a smooth curve in the boundary of the inducing element. The DP displays were generated by placing the circles a slight distance from the $D_{1}\left(0.5 \mathrm{~cm} ; 0.2^{\circ}\right.$ visual angle $)$. The addition of identical circles to both DA and DP displays ensured equality of size of the inducing elements in the DA/DP pairs in Figure 2. For the DA and DP pair in the circle display (Figure 3), the approximate size of the inducing elements was not the same for both DP and DA displays (the inducing elements in the DA display were larger). The DA display shown in Figure 3 was formed by placing 12 circles (radius $=1 \mathrm{~cm} ; 0.4^{\circ}$ visual angle) at equal intervals around a circle of radius $4 \mathrm{~cm}$. The DP display (right side of Figure 3) was formed by removing parts of each of the circles in the DA display, resulting in the presence of $D_{1} s$ in each of the inducing elements.

The subjective figure displays were all presented on the left side of the monitor. On the right side, a solid blue figure was presented.

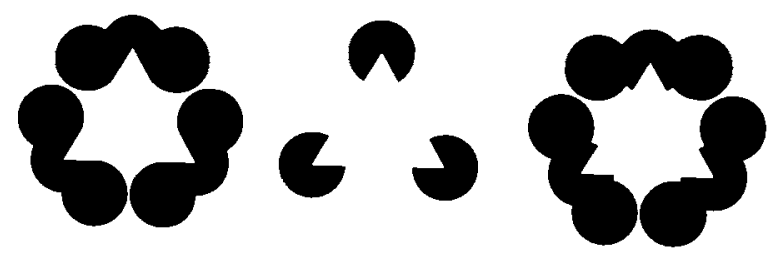

Figure 2. Display types in Experiment 1. The central display is the base subjective triangle display. The $D_{1} s$ in the base display were removed by adding circles tangent to the edges of the subjective triangle. The discontinuity-absent display is on the left. Circles were also added to the base display to generate the discontinuity-present display, shown on the right, but the additional circles were not tangent to the subjective triangle.
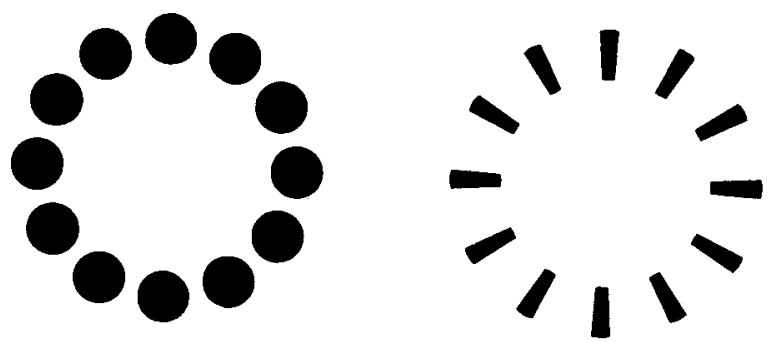

Figure 3. Circle displays used in Experiment 1. Twelve circles equidistant from one point form the discontinuity-absent display (left); parts of each circle are removed to form the discontinuitypresent display (right). 


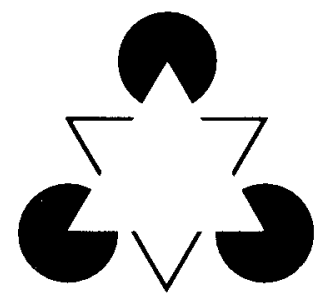

a)

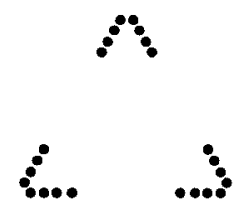

b)

Figure 4. Modulus figures used to anchor the subjective figure rating scale. (a) The first of four displays used to explain subjective figures to subjects. In all of the experiments that required clarity ratings, subjects were told to rate any subjective figure as strong as this one a "10." (b) The second of four displays used to explain subjective figures to subjects. The display used to explain the difference between seeing a subjective figure (Figure 4a) and imagining a triangle that fit between the points in this display.

This figure was identical in shape and orientation to the subjective figure that we expected the subjects to see in the particular subjective figure display.

Procedure. For all experiments, the subjects were seated in front of the monitor and given the following instructions:

This is an experiment involving subjective figures. I will show you some displays that contain subjective figures and some displays that do not contain subjective figures. [The subject was then shown Figure $4 \mathrm{a}$; the experimenter pointed to the display and indicated the outer boundaries of the subjective triangle.] This is an example of a subjective figure; the central triangle is the subjective figure. It is important to note two of its properties. First, you can see the entire triangle, and second, you can see a clear edge even in the areas between the white figures in the display. [The experimenter then displayed Figure $4 \mathrm{~b}$, and pointed to the area between the white dots.] This is not a subjective figure. While you can imagine a triangle formed by the three sets of dots, most people do not see a complete figure with clear edges between the white areas. Because the display does not contain a triangle with clear edges, it does not contain any subjective figure.

These instructions were repeated, using a subjective figure display containing a subjective rectangle and a similar display in which only the outlines of the inducing elements were shown. (Such outline inducing-element displays do not produce subjective figures.) The subject was then asked if he or she understood what was meant by a "subjective figure." If the subject answered "no," the experimenter showed the displays a second time. The experimenter then explained the subject's task as follows:

You will now see several displays similar to the displays that you have just seen. We would like you to indicate whether or not you see a subjective figure in the display, but only say "yes, I see a figure" if that figure matches the blue figure on the right side of the screen in both size and shape. If you do see a subjective figure, we would like you to rate the strength of the figure using a scale from 1 to 10 . If the figure is as clear as the first figure that you saw, you should rate it a 10; if it is less clear, you should give it a lower number. Do you have any questions?
Before the experimental displays were presented, 10 practice displays were presented, which allowed the subject to become comfortable with rating subjective figure displays and to ask any questions. We included two standard subjective figure displays in the set of practice displays. One of the two standards was a subjective circle formed from radiating lines, a figure that other investigators have found to produce clear subjective figures (Petry, Harbeck, Conway, \& Levey, 1983); the other standard was a subjective square formed by placing crosses at each corner, a figure that produces weak or no subjective figures (Day \& Kasperczyk, 1983). The subjects' responses to these figures were to be used to screen out any subjects who misunderstood the task or had unusual response tendencies. The criteria were that the radiating lines display should be rated no lower than 6 , and the cross display no higher than 2 .

\section{Results}

No subjects were excluded from the analysis on the basis of their ratings of the displays shown in the practice trials. (All subjects rated the subjective circle with radiating inducing elements 6 or higher and the display with cross-shaped inducing elements 2 or lower.)

By assigning responses of "no subjective figure" a rating of 0 , we calculated the mean clarity ratings for each figure; these are presented in Table 1. A two-way repeated measures ANOVA with type of subjective figure and presence/absence of $D_{1} s$ as factors revealed a main effect of presence versus absence of $D_{1} s[F(1,23)=20.80$, $p<.0001]$ and a main effect of display $[F(3,69)=3.89$, $p<.01]$. There was also a significant interaction between display and presence/absence of $D_{1} \dot{s}[F(3,69)=7.89$, $p<.00011$. This interaction was primarily due to the stimulus pair whose potential subjective figure was a circle (Figure 3). Its DA member obtained the lowest rating $(M=1.87)$, whereas its DP member obtained the highest rating of any of the displays $(M=6.21)$. When these two ratings were removed from the analysis, there was no longer a significant interaction $(F<1)$.

All DP displays were reliably rated higher than their corresponding DA displays were. Planned comparisons between the pairs of ratings for the triangle (Figure 2), square, rounded triangle, and circle (Figure 3 ) gave $F(1,69)$ values of $7.55,9.43,6.69$, and 70.67 , all ps $<.01$.

\section{Discussion}

The presence of $D_{1}$ s had a strong effect on the subjects' perception of subjective figures. In all four cases, the sub-

Table 1

Mean Subjective Figure Ratings for Experiment 1

\begin{tabular}{lcc}
\hline Subjective Figure & Discontinuity & Mean Rating \\
\hline Triangle & present & 4.92 \\
& absent & 3.96 \\
Square & present & 5.46 \\
& absent & 4.12 \\
Rounded triangle & present & 3.96 \\
& absent & 2.37 \\
Circle & present & 6.21 \\
& absent & 1.87 \\
\hline
\end{tabular}


jective figure seen in the DA displays was rated lower than those seen in the DP displays. Across all four DP/DA pairs, $67 \%$ of the subjects were more likely to rate DP higher than DA; $12 \%$ were more likely to rate DA higher than DP.

Although differences between DP and DA displays were clear, clarity ratings were generally rather low. It is important to note that the displays used here were not developed to optimize subjective figure perception, but only to make comparisons regarding the role of $D_{1} s$. For example, it is known that smaller gap size and larger extents of physically specified edges produce clearer visual interpolation (Shipley, 1988; Shipley \& Kellman, 1988). Specifically, the ratio of interpolated edge to the entire edge is a good predictor of subjective figure clarity (with higher ratios giving lower clarity). The generally low ratings in the present study stem in part from the high ratios employed. Displays with such high ratios were required by the size of the circles used to round off the $D_{1} s$. The inducing elements had to be spaced sufficiently far apart so that the circles used to round off the $D_{1} s$ did not overlap.

Virtually all previous examples of subjective figure displays have used patterns with sharp corners or $D_{1} s$. The present study provides the first experimental evidence that such discontinuities influence the perception of subjective figures. The greater subjective figure clarity of the DP displays accords with our claim that $D_{1}$ s are necessary for the perception of subjective figures, but the fact that the DA displays received a nonzero clarity rating is potentially problematic. The implications of these results for a unit formation theory based on $D_{1} s$ are taken up in the General Discussion section below.

Such a discussion, however, would be premature without at least two further studies: (1) a demonstration that the differences found in Experiment 1 were not due to some artifact resulting from the particular paradigm, and (2) a demonstration that these differences were not due to the specific displays used. Experiment 2 was addressed to the first concern, and Experiments 3 and 4 to the second.

\section{EXPERIMENT 2}

In Experiment 1, subjects were purposely shown the form of the figure they should be rating. There are several potential problems with this procedure. Subjects might have reported a subjective form that corresponded to the blue figure because that blue figure was present; subjects' ratings might have reflected suggestion or set produced by the standard. Furthermore, some of the displays might have produced a subjective figure other than the blue figure, which would have resulted in a rating of 0 for that display. Neither of these potential problems provide any explanation for the differences between the DP and DA displays. Nevertheless, to determine the robustness of these findings, Experiment 1 was replicated without standards. A new group of subjects was shown displays iden- tical to those used in Experiment 1. Instead of being asked to rate the strength of the figure, the subjects were merely asked to make presence/absence judgments about subjective figures in each display. When a subjective figure was reported, the subjects were asked to describe the subjective figure to confirm that the blue figures used in Experiment 1 were indeed the figures subjects would spontaneously report.

\section{Method}

Procedure. The procedure for Experiment 2 differed from the procedure described for Experiment 1 in the following ways: The instructions used were the same as those used in Experiment 1, except that the subjects were told to report whether or not they saw a subjective figure. If they did see a subjective figure, they were asked to draw it.

Twenty undergraduate males and females who received $\$ 2.50$ for a 30-min session served as subjects for this experiment.

Stimuli. The stimuli for this experiment were the four pairs of stimuli used in Experiment 1.

\section{Results}

No subjects were excluded from the analysis.

The results of Experiment 2 mirror those obtained in Experiment 1. For all four stimulus pairs, the number of subjects who reported a subjective figure was considerably greater for the DP than for the corresponding DA display. Table 2 shows the number of subjects reporting a subjective figure for each of the displays. Chi-squares for each pair of DA-DP displays $(d f=1, n=20)$ were: triangle, $14.5(p<.01)$; square, $5.0(p<.05)$; rounded triangle, $3.1(p=.07)$; and circle, $20.4(p<.01)$.

All subjects who reported seeing a subjective figure reported figures that matched the shape of the blue figure used in the corresponding display from Experiment 1.

\section{Discussion}

Experiment 2 confirmed the findings of Experiment 1. Subjects were more likely to report a subjective figure in DP than in DA displays. These results more clearly suggest the necessity of discontinuities for visual interpolation. The largest number of subjects (out of 20) reporting subjective figures in any DA display was 5 . The results also suggest that the difference in rating between DA and DP displays found in Experiment 1 was not due to the

Table 2

Number of Subjects Reporting a Subjective Figure for Experiment 2

\begin{tabular}{lcc}
\hline Subjective Figure & Discontinuity & $\begin{array}{c}\text { Number of } \\
\text { Subjects }\end{array}$ \\
\hline Triangle & present & 17 \\
& absent & 5 \\
Square & present & 12 \\
& absent & 5 \\
Rounded triangle & present & 8 \\
& absent & 3 \\
Circle & present & 15 \\
& absent & 1 \\
\hline
\end{tabular}


use of a standard figure or to magnitude estimations of clarity. Finally, the subjects' ratings of subjective figure strength in Experiment 1 corresponded well with the number of subjects who reported a subjective figure, suggesting that clarity ratings and probability of report depend on the same perceptual representations.

\section{EXPERIMENT 3}

The stimuli used in Experiments 1 and 2 contained simple geometric subjective figures. Rock (1983), as well as others (Buffart, Leeuwenberg, \& Restle, 1981; van Tuijl \& Leeuwenberg, 1982; Wallach \& Slaughter, 1988), has suggested that familiarity and regularity are important to the perception of subjective figures. According to Rock, the possibility that an inducing element can be completed to form a familiar or regular figure is responsible, at least in part, for the system hypothesizing the presence of, and therefore seeing, a subjective figure. Both Rock (1983) and Wallach and Slaughter (1988) emphasize familiarity of a subjective figure as an important determinant in perceiving the figure.

Buffart et al. (1981) proposed that unit formation is a by-product of a perceptual system that uses a minimal code for the encoding of all scenes. In cases where a partially occluded figure is seen, it is seen because the partially occluded figure requires a smaller code than the visible parts. Consider Figure 5a. According to coding theory, two overlapping rectangles are seen because encoding the display as two rectangles (Figure $5 b$ ) requires less code than encoding it as one rectangle and an L-shaped figure

a)

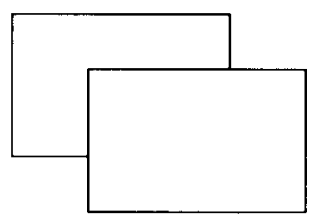

b)

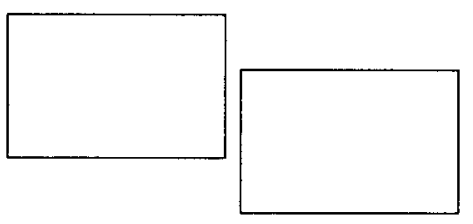

c)

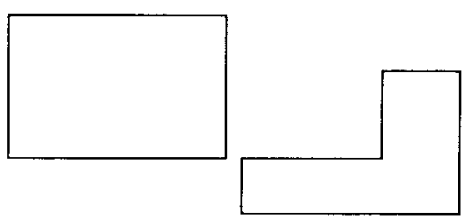

Figure 5. Illustration of coding theory. Two possible perceptual interpretations of the figure seen in (a) are shown in (b) and (c). (See text.)
(Figure 5c). The difference in code size between Figures $5 \mathrm{~b}$ and $5 \mathrm{c}$ is due to the symmetry of a rectangle. Van Tuijl and Leeuwenberg (1982) presented a coding theory account for the perception of subjective figures. They suggested that a subjective figure will be seen whenever the code necessary to encode the inducing elements as figures extending under the subjective figure is smaller than the code necessary to encode the inducing elements as complete figures. In the case of a display like that in Figure 4a, encoding the inducing elements as circles is more efficient than encoding them as five sixths of a circle; hence, a subjective triangle is seen.

The model of unit formation that we have proposed (Kellman \& Shipley, in press) explicitly denies that either regularity or familiarity of either the subjective figure or the completed form of the inducing elements is necessary for subjective figure perception. In Experiment 3, we investigated the effect of presence and absence of $D_{1} s$ in displays with irregular subjective figures and inducing elements that cannot be completed as symmetrical, regular figures.

\section{Method}

Procedure. The procedure used in Experiment 3 was the same as the one described for Experiment 1, except that the displays did not contain any blue figures, and subjects were asked to report and rate the clarity of any perceived subjective figure.

Because agreement between the two methods of assessing subjective figures used in Experiments 1 and 2 was very high, we combined both methods. We asked subjects for magnitude ratings but did not provide a standard against which the subject had to compare their percepts, asking them instead to draw the subjective figures that they saw. This procedure avoids any concerns that the standard influences the percept, and it provides subjects with a way to make finer discriminations than are allowed by a yes/no judgment.

Twenty undergraduate males and females who received $\$ 2.00$ for a 30 -min session served as subjects for this experiment.

Stimuli. Five pairs of DA-DP displays were constructed for Experiment 3 . In each pair of displays, the inducing elements were arrayed so that potential subjective figures formed by connecting the specified edges would be irregular. One of the DA-DP pairs is shown in Figure 6. Four of the five pairs resembled this display in that the DA display was constructed by smoothing the $D_{1} s$ present in the DP display. The fifth pair (shown in Figure 7) was similar to that in Figure 3 (in Experiments 1 and 2), in that the inducing elements in the DA display consisted of semicircles tangent to the potential subjective figure (the semicircles were extended away from the central region to form irregular inducing elements). As in Figure 3, the DP display (on the right in Figure 7) was created by removing parts of the inducing elements in the DA display so that $D_{1} s$ were formed.

\section{Results}

No subjects were excluded from the analysis on the basis of their ratings of the displays shown in the practice trials. (All subjects rated the subjective circle with radiating inducing elements 6 or higher and the display with cross-shaped inducing elements 2 or lower).

Mean clarity ratings for each figure are presented in Table 3. A two-way repeated measures ANOVA with subjective figure type and presence/absence of $D_{1} s$ as factors revealed a main effect of presence/absence of $D_{1} s$ 

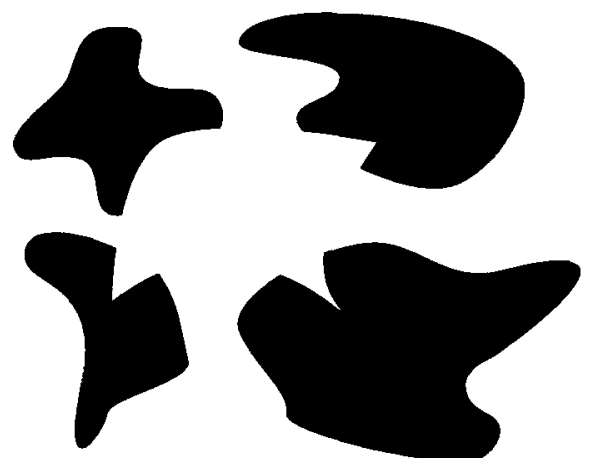

DP Display

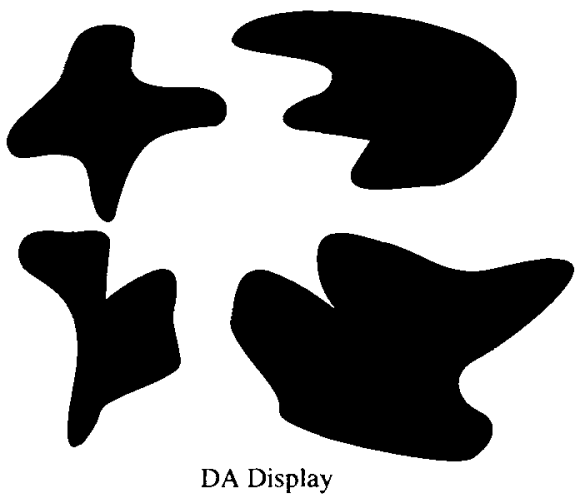

Figure 6. An example of the discontinuity-absent (DA) and discontinuity-present (DP) displays used in Experiment 3. Unfamiliar figures were used for the subjective figure and the inducing elements. The DA display was created by rounding off the corners present in the DP displays.
$[F(1,19)=112.66, p<.0001]$ and a main effect of display $[F(4,76)=6.43, p<.0002]$. There was also a significant interaction between display and presence/absence of $\mathrm{D}_{1} \mathrm{~s}[F(4,76)=7.64, p<.0001]$. Subsequent comparisons indicated that this interaction was primarily due to the stimulus pair shown in Figure 7; its DA member was given the lowest rating $(0.55)$, whereas its DP member obtained the second highest rating (4.65) of any of the displays. When these two ratings were removed from the analysis, there was no longer a significant interaction $[F(3,57)=1.07, p>.25]$.

All DP displays were reliably rated higher than were the corresponding DA displays. On the average, the DP displays were given twice the clarity rating of the DA displays. And, all of the subjects were more likely to rate DP displays higher than the corresponding DA display. In fact, only 5 times, out of 100 , did a subject rate a DA display higher than the corresponding DP display. Planned comparisons between corresponding DA and DP displays produced $F(1,76)$ values of $8.53,8.53,15.78,24.08$, and 91.79 , all $p s<.005$. The first and last $F$ values are the planned comparisons for the displays shown in Figures 6 and 7 , respectively.

\section{Discussion}

The results of this experiment confirm the importance of $D_{1} s$ in the perception of subjective figures. The effect of $D_{1} \mathrm{~s}$ is even clearer in Experiment 3 than it is in Experiment 1 . It is possible that suggesting the central figure in Experiment 1 enhanced reports for the DA displays (cf. Wallach \& Slaughter, 1988). Clearly, the effect of $D_{1} s$ is not limited to displays in which the subjective figure

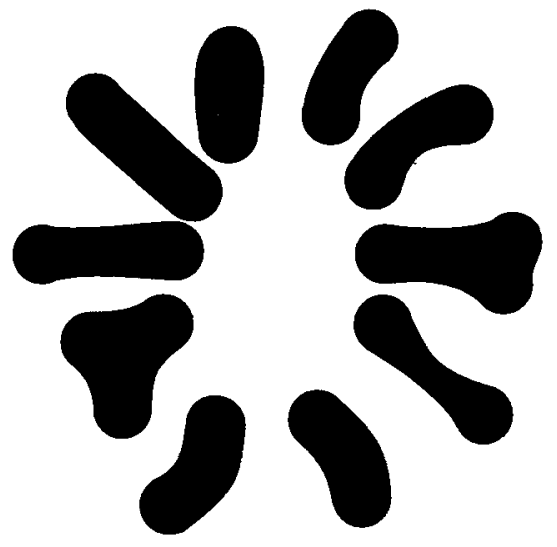

DA Display

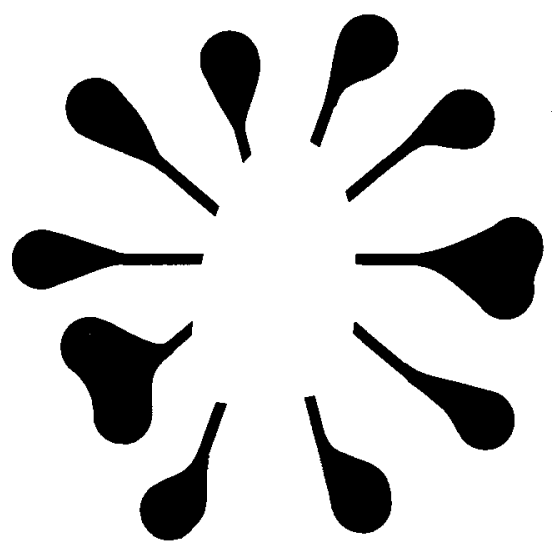

DP Display

Figure 7. The discontinuity-present display (DP), which was created by removing parts of the discontinuity-absent display (DA), from Experiment 3. 
Table 3

Mean Subjective Figure Ratings for Experiment 3

\begin{tabular}{ccc}
\hline Subjective Figure & Discontinuity & Mean Rating \\
\hline 1 & present & 3.10 \\
& absent & 1.85 \\
2 & present & 4.90 \\
& absent & 3.65 \\
3 & present & 4.15 \\
& absent & 2.45 \\
4 & present & 3.65 \\
& absent & 1.55 \\
5 & present & 4.65 \\
& absent & 0.55 \\
\hline
\end{tabular}

and the potential completion of the inducing elements are familiar or regular figures. Again, the clarity ratings of all of the displays are relatively low, and again this is presumably due to the spacing of the inducing elements required to allow the $D_{1} s$ to be removed in the DA displays.

This experiment also adds to the growing set of observations (Kanizsa, 1979; Kellman \& Shipley, in press) and experiments which show that regularity and familiarity are not necessary features of subjective figure displays.

\section{EXPERIMENT 4}

Experiments 1, 2 and 3 provide clear support for the claim that $D_{1} s$ are an important feature of subjective figure displays. But they do not provide unconditional support for the claim that $D_{1} s$ are necessary for the perception of subjective figures. In Experiments 1 and 3, over half the subjects gave ratings greater than 0 for DA displays. In Experiment 2, about a quarter of the subjects reported a figure in the DA displays. One possible account for these results is that $D_{1} s$ are not necessary for the perception of subjective figures; they are simply one factor that affects the strength of subjective figures. Some researchers (Day, 1987; Halpern, 1981) have suggested that the perception of subjective figures is multiply determined; perhaps $D_{1} s$ are simply one such determining feature.

If $D_{1} s$ are simply one of many features affecting the clarity of subjective figures, then the effect of $D_{1} s$ and the effect of any other feature should be additive. If, on the other hand, $D_{1} s$ are necessary for the perception of subjective figures, then the effect of $D_{1} s$ will not be additive; rather, the effect of any feature should only occur when $D_{1}$ s are present.

In Experiment 4, we tested the effects of two parametric variations on displays in which $D_{1} s$ were present or absent. The two parameters were the number and length of the inducing elements. The first of these was chosen on the basis of work done by Petry, Harbeck, Conway, and Levey (1983), who reported that subjects rated displays with more inducing elements higher than displays with fewer inducing elements. The second parameter was based on some of our own pilot studies. If $D_{1} s$ are indeed necessary, both inducing elements' size and their num- ber should affect subjective figure perception in DP displays but not in DA displays. These two parameters were varied in displays similar to those shown in Figures 3 and 7. This type of display was chosen because $D_{1} s$ are most clearly absent in the DA displays.

\section{Method}

Procedure. The procedure in Experiment 4 was the same as that in Experiment 3.

Thirty undergraduate males and females who received $\$ 2.50$ for a 30-min session served as subjects for this experiment.

Each of the 30 subjects was presented with 18 stimuli twice. In the first block of 18 trials, all 18 stimuli were presented in a random order; in the second block of trials, the same 18 stimuli were presented in a different random order.

Stimuli. All of the displays contained inducing elements placed at equal intervals around the center of the screen. The potential subjective figure that these displays might produce was a subjective circle with a radius of $3.4 \mathrm{~cm}$ ( $1.3^{\circ}$ visual angle). The displays were generated by factorially combining three different numbers of inducing elements $(8,10$, or 12$)$, three different sizes of the inducing elements (small, medium, or large), and presence versus absence of $D_{1} s$ on the inducing elements, for a total of 18 different displays. The central displays in Figure 8 are the 10 medium-length inducing elements without $D_{1} s$ and 10 medium-length inducing elements with $D_{1} s$. The left half of Figure 8 shows the DA displays, the right half the corresponding DP displays. The top row gives examples of displays with 12 long inducing elements. The bottom row shows examples of displays with 8 short inducing elements.

The selection of the number of inducing elements was limited by the minimum number necessary to achieve a reasonably clear subjective circle (8 elements) and the maximum number of DA inducing elements that would fit around the circle without overlapping ( 12 elements). The third number was simply the midpoint between the limits. The length of the short inducing elements was determined by the diameter of the circle used for the short DA inducing element $\left(2 \mathrm{~cm}\right.$; visual angle $\left.0.8^{\circ}\right)$. The length of the long inducing elements was determined by the length of the DP inducing element necessary to produce a figure with the same area as the short DA inducing element $\left(4.6 \mathrm{~cm}\right.$; visual angle $\left.1.8^{\circ}\right)$. Again the medium value selected was the midpoint between the two extremes $\left(3.3 \mathrm{~cm}\right.$; visual angle $\left.1.3^{\circ}\right)$. The short DA inducing elements were circles of radius $=1 \mathrm{~cm}$ (visual angle $0.4^{\circ}$ ). The longer inducing elements were thick lines of width $=2 \mathrm{~cm}$ (visual angle $0.8^{\circ}$ ) with semicircles of radius $=1 \mathrm{~cm}$ (visual angle $0.4^{\circ}$ ) at both ends of each line. The width of the part of the DP inducing elements tangent to the subjective circle was $0.4 \mathrm{~cm}$ (visual angle $0.2^{\circ}$ ). This length was determined by the length of the edge on the DA inducing elements that would have been in contact with the subjective circle. The sides of the DP inducing elements were created by extending a line perpendicular to the subjective circle. The end away from the subjective circle was a semicircle, which was added to avoid having that end of the inducing elements produce subjective contours.

Dependent measures and data analysis. Prior to running the experiment, it was decided that subjects who did not give reliable ratings would be excluded from the analysis. There were three criteria of reliability. The first involved responses to a display known not to produce subjective contours, which was presented during the first 10 warm-up trials. (See procedure section of Experiment 1.) If a subject gave a rating of 2 or higher to this display, he or she was excluded. One subject was excluded for this reason. The second criterion was based on consistency in reporting whether or not a figure was seen. If on four or more pairs of presentations of each display a subject reported seeing a subjective figure on one and only one of the two occasions, he or she was excluded. Two subjects 


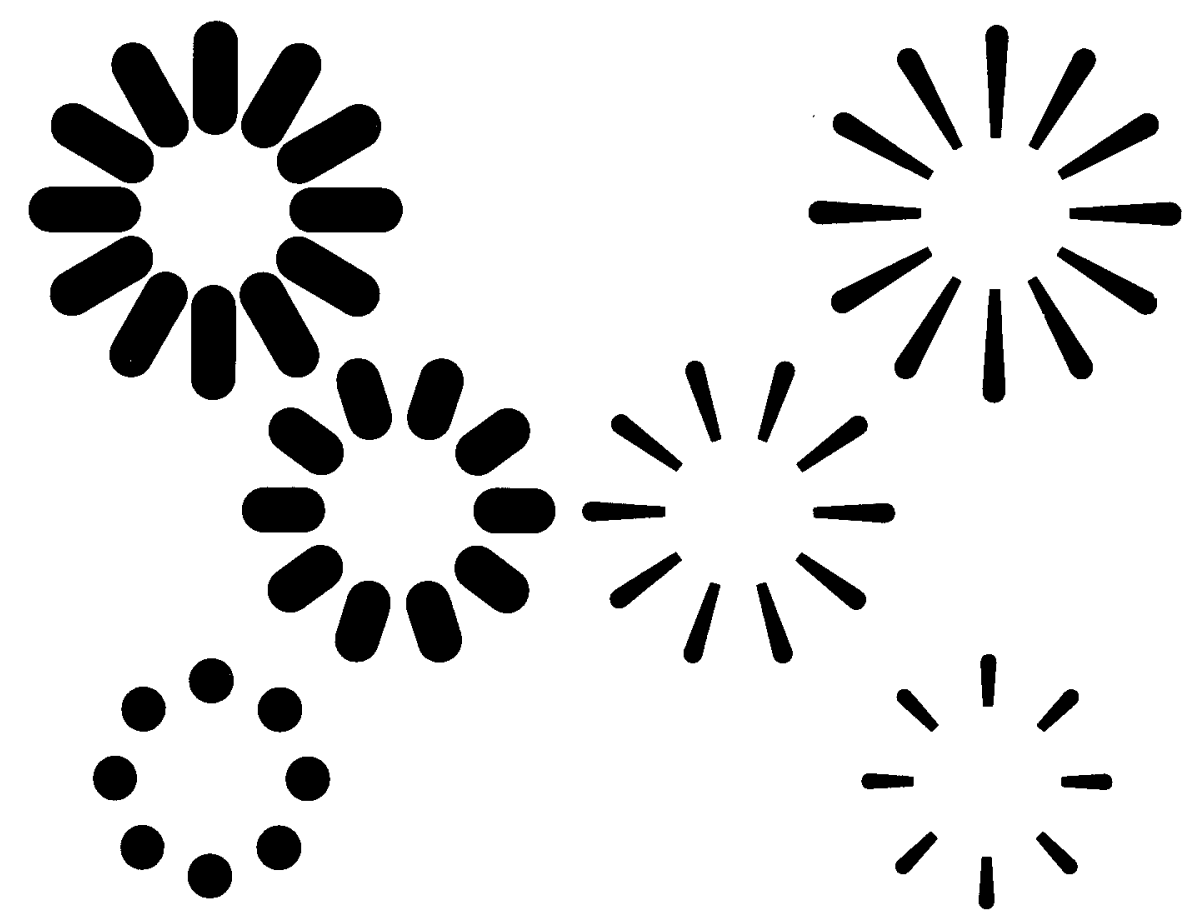

Figure 8. Six of the displays used in Experiment 4. The three displays on the left are examples of the discontinuity-absent displays; the three displays on the right are examples of the discontinuitypresent displays. The displays at the lower left and lower right contain 8 short inducing elements. The displays in the center have 10 medium inducing elements. The upper left and upper right displays have 12 long inducing elements.

were excluded on this basis. The final criterion was based on the consistency in ratings for a given display between the first and second presentations. If a subject's two ratings of two or more displays differed in magnitude by 5 or more, he or she was excluded. Seven subjects were excluded on this basis. It was necessary to collect data from 30 subjects to find 20 subjects who met all three of the reliability criteria.

Because such a large number of subjects were rejected, all analyses were conducted twice: once including and once excluding the 10 subjects who were rejected. The two sets of analyses gave virtually identical results. All analyses reported here are based on the final set of 20 subjects.

\section{Results}

Table 4 shows the mean subjective figure ratings for each type of display; as in Experiment 1, means were calculated by assigning a rating of 0 to responses of "no subjective figure." Because this experiment focused on the comparison of DA and DP displays, analyses were restricted to trials in which subjects reported seeing a subjective circle. (In $1.8 \%$ of the displays, subjects reported seeing a subjective star; the percentage over all 30 subjects was 3.9.)

Inspection of Table 4 reveals that all three factors had powerful effects on subjective figure ratings. These impressions were confirmed by results of a four-way repeated measures ANOVA with number of inducing elements, size of inducing elements, presence/absence of $D_{1} s$, and repetition as the factors.
The most important factor was the presence versus absence of $D_{1} s$. There was a main effect of presence versus absence of $\mathrm{D}_{1} \mathrm{~s}[F(1,19)=69.2, p<.0001]$. The mean rating for the DP displays was 4.13 , whereas the mean rating for the DA displays was .003 . Displays without

Table 4

Mean Subjective Figure Ratings for Experiment 4

\begin{tabular}{cllcc}
\hline \multicolumn{2}{c}{ Inducing Elements } & & \\
\cline { 1 - 2 } Number & Size & & Discontinuity & Mean Rating \\
\hline 8 & small & & present & 2.77 \\
& medium & & present & 3.05 \\
& long & & present & 3.42 \\
& small & & present & 3.67 \\
& medium & present & 4.17 \\
& long & present & 4.52 \\
12 & small & present & 5.05 \\
& medium & present & 5.15 \\
& long & present & 5.52 \\
\multirow{2}{*}{8} & small & absent & 0.02 \\
& medium & absent & 0 \\
& long & absent & 0 \\
10 & small & absent & 0 \\
& medium & absent & 0 \\
& long & absent & 0 \\
& small & absent & 0 \\
& medium & absent & 0 \\
& long & absent & 0 \\
\hline
\end{tabular}


Table 5

Mean Subjective Figure Ratings as a Function of Number of Inducing Elements in Experiment 4

\begin{tabular}{ccc}
$\begin{array}{c}\text { Number of } \\
\text { Inducing Elements }\end{array}$ & Discontinuity & Mean Rating \\
\hline 8 & present & 3.08 \\
10 & present & 4.12 \\
12 & present & 5.24 \\
8 & absent & .008 \\
10 & absent & 0 \\
12 & absent & 0 \\
\hline
\end{tabular}

Table 6

Mean Subjective Figure Ratings as a Function of Inducing Element Size in Experiment 4

\begin{tabular}{ccc}
\hline $\begin{array}{c}\text { Size of } \\
\text { Inducing Elements }\end{array}$ & Discontinuity & Mean Rating \\
\hline short & present & 3.80 \\
medium & present & 4.10 \\
long & present & 4.49 \\
short & absent & 0 \\
medium & absent & 0 \\
long & absent & 0 \\
\hline
\end{tabular}

$D_{1} s$ were virtually never perceived as having subjective figures. There were also main effects of number of inducing elements $[F(2,38)=57.1, p<.0001]$ and size of the elements $[F(2,38)=11.2, p<.0001]$. The subjects did not rate the displays differently in the two blocks $(F<1)$.

Table 5 shows the mean ratings for displays with 8,10 , and 12 inducing elements for both DP and DA displays. There is a reliable effect of number of inducing elements in the DP displays but none in the DA displays. The same pattern can be seen in Table 6, which shows the mean ratings for DP and DA displays with short, medium, and long inducing elements. There is a clear effect of size in the DP displays but no such effect for DA displays. An ANOVA yielded large interactions between the number of inducing elements and the presence/absence of $D_{1} s$ $[F(2,38)=58.6, p<.0001]$ and between the size of inducing elements and the presence/absence of $\mathrm{D}_{1} \mathrm{~s}[F(2,38)$ $=11.7, p<.0001]$. No other interactions reached significance (all $p \mathrm{~s}>.15$ ).

Planned comparisons supported these observations. Within the DP displays, subjects rated the displays with 12 inducing elements $(M=5.24)$ higher than the displays with 10 inducing elements $[M=4.09 ; F(1,38)=64.5$, $p<.0001]$ and with 8 inducing elements $[M=3.05$; $F(1,38)=232.6, p<.0001]$. Displays with 10 inducing elements were rated higher than were displays with 8 inducing elements $[F(1,38)=51.1, p<.0001]$. In the DA displays, the mean ratings were $.008,0$, and 0 for the displays with 8,10 , and 12 inducing elements, respectively (all $F \mathrm{~s}<1$ ).

The same pattern of results is found for the interaction between the presence/absence of $D_{1} s$ and the size of inducing elements. Table 6 presents the mean ratings for displays with short, medium, and long inducing elements for DA and DP displays. Within the DP displays, the subjects rated displays with long inducing elements $(M=$ 4.49) higher than displays with medium inducing elements $[M=4.10 ; F(1,38)=14.6, p<.0005]$ and with short inducing elements $[M=3.80 ; F(1,38)=45.7$, $p<.0001]$. They rated displays with medium inducing elements higher than displays with short inducing elements $[F(1,38)=8.60, \mathrm{p}<.006]$. In the DA displays, subjects did not rate displays with long inducing elements $(M=0)$ higher than displays with medium inducing elements $(M=0)$ or with short inducing elements $(M=$ 0.008 ; all $F \mathrm{~s}<1$ ).

\section{Discussion}

In Experiment 4, we tested the necessity of $D_{1} s$ by examining the relationship between the presence of $D_{1} s$ and two other factors known to influence the perceived clarity of a subjective figure. Although all three factors had clear effects, $D_{1} s$ did not act additively in conjunction with the number and size of inducing elements. The effects of number and size were seen only in displays that contained $\mathrm{D}_{1} \mathrm{~s}$. This finding strongly supports the hypothesis that $D_{1} s$ are necessary for the perception of subjective figures. First derivative discontinuities are not simply one of a number of factors that add together to determine the strength of subjective figures. There is no way to trade off the presence of $D_{1} s$ by increasing the value of other variables, such as number of inducing elements. In the absence of $D_{1} s$, these other variables have no effect, suggesting that $D_{1} s$ are a prerequisite for subjective figure perception.

\section{GENERAL DISCUSSION}

Are $D_{1} s$ necessary for the perception of subjective figures? Subjects report clearer subjective figures in displays containing $D_{1} s$ than in displays without such discontinuities. Subjects are also far more likely to report seeing subjective figures in displays that contain $D_{1} s$. Finally, parametric variations in subjective figure displays only appear to influence the clarity and probability of reporting a subjective figure if the display contains $D_{1} s$.

If $D_{1} s$ are necessary, why do some subjects report seeing a subjective figure in displays without $D_{1} s$ ? Experiment 4 rules out the simple account of this finding; it is not due to $D_{1}$ s having an additive effect on subjective figure perception. There are a number of other possible explanations.

1. False perceptual report. The subjects did not see any subjective figures in DA displays but reported weak subjective figures due to the similarity of the displays to other displays in which they did see subjective figures. An attempt was made to reduce the chance of this occurring by emphasizing the importance of reporting "no subjective figure" when there was no clear figure.

2. Stimulus limitations. The resolution of the monitor was not high enough to eliminate $D_{1}$ s completely. Due to the digital nature of the equipment, all displays con- 
tained $D_{1} s$. Perhaps the jagged nature of the curves in the DA displays was sufficient to produce weak subjective figure ratings. This seems fairly unlikely, because the DA displays in Experiment 4, which elicited clarity ratings of approximately 0 , were generated in the same way as the displays in Experiments 1, 2, and 3.

3. Generalization of discontinuity. It is important to note that the visual system's use of $D_{1} s$ must involve thresholds. We would expect some curves with extremely small radii of curvature to function perceptually as $D_{1} s$ despite their having technically continuous first derivatives. Likewise, some boundaries between two connecting edges of similar orientation may be mathematically discontinuous in slope but will be mistaken for smooth. The reason for these expectations is that classes of functions exist that contain $D_{1} s$ but that can be arbitrarily smooth in appearance. Conversely, classes of functions exist that do not have first derivative discontinuities but can be made arbitrarily sharp in curvature. Exactly which curves are classified as $D_{1} s$ by the visual system has not been fully determined. Perhaps the curves used in some of the DA displays fall within the range of curves that are treated as being $D_{1} s$. The present research provides basic evidence for the role of $D_{1} s$; future research will take up the issue of thresholds systematically.

4. Incomplete characterization of discontinuity. The final possibility is that the presence of discontinuities in the first derivative of a function is not the only condition that initiates unit formation. Perhaps other sources of information can be used to identify overlapping boundaries. A comparison of the DA displays that resulted in some unit formation with those that produced little or no unit formation suggests one additional source of information. ${ }^{3}$ The DA displays that produced the strongest ratings of subjective figure clarity were the displays like the ones shown in Figures 2 and 6. Such displays have discontinuities in the second derivative. Either the inducing elements have a straight contour that joins a curved contour (as, e.g., in Figure 2), or they have a contour curving in one direction joined to a contour of differing curvature (as, e.g., in Figure 6). In the DA displays that produced very weak contour clarity ratings, there is no clear, straight contour joining a curved contour, or curved contour joining a differently curved contour (Figures 3, 7, and all of the DA displays used in Experiment 4). When a contour changes from flat to curved, its first derivative changes from constant to increasing (or decreasing). At that point, the second derivative changes, discontinuously, from 0 to finite. Perhaps both discontinuities in the first and second derivatives are initiating conditions for interpolation.

Changes in the second derivative could be useful as a source of information in cases where objects, of a similar color, overlap but have indistinguishable slopes at the point of intersection in their projections. Figure 9 illustrates such a case. The need for such information may be small, since such special cases could also be disambiguated by moving one's viewpoint slightly, thereby disrupting the fortuitous alignment of contours. Moreover, when two objects of differing surface quality are involved (in contrast to Figure 9), there will always be $\mathrm{D}_{1}$ in the projection.

Our data suggest that discontinuities in the second derivative are not as effective as those in the first derivative. In Experiments 1, 2, and 3, ratings were weak and "presence" reports were few for displays containing second derivative discontinuities without first derivative discontinuities. Only when first derivative discontinuities are present do subjects give strong clarity ratings and frequent reports of subjective figures. We are currently investigating whether the units seen in the DA displays are a result of generalization from first derivative discontinuities or due to second derivative discontinuities.

\section{Implications for Perception of \\ Partially Occluded Figures}

The importance of $D_{1} s$ is utilized in a more general model of unit formation proposed by Kellman and Shipley (in press). Although presentation of the approach is beyond the scope of this paper, several more general implications of the present findings are worth noting. The Kellman and Shipley theory provides an explanation for the perception of boundaries in the absence of local physical specification. In natural environments, the most important application of this theory is to the perception of

a)
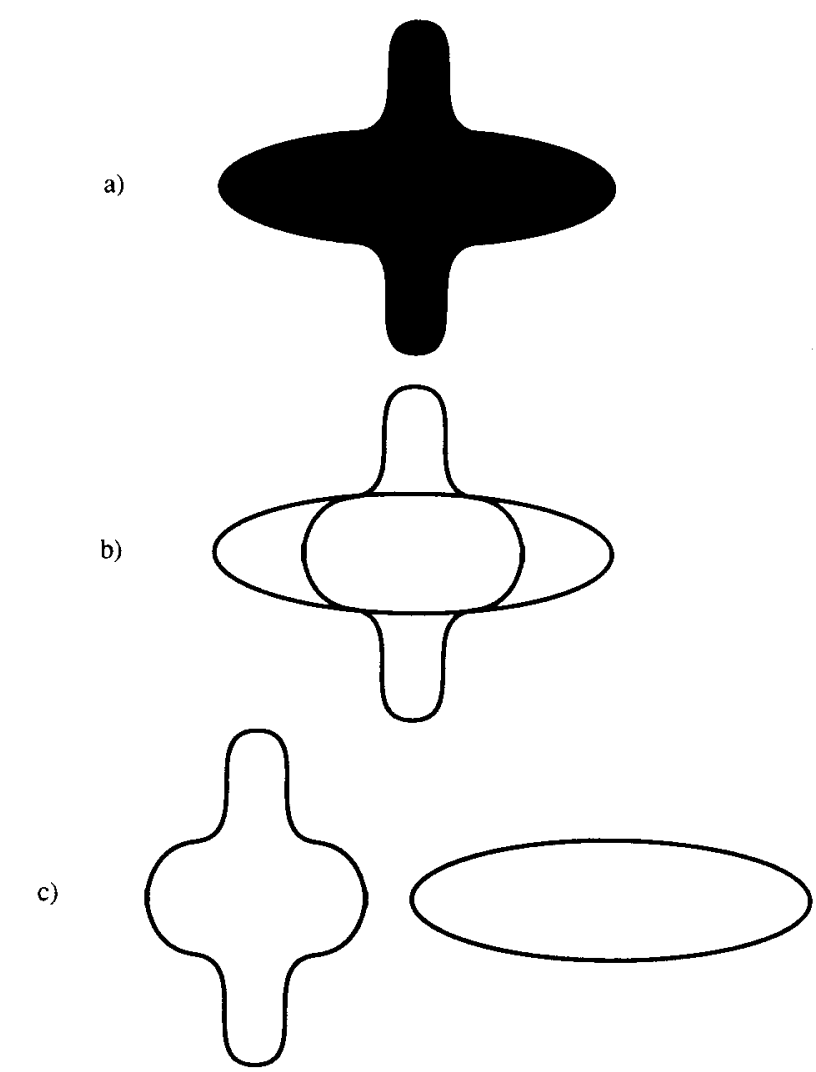

Figure 9. Example of second derivative discontinuity. (a) An example of two overlapping figures where there is a discontinuity in the second derivative of the contour but no discontinuity in the first derivative. (b) A line drawing illustrating the boundaries of the two units. (c) The two separate units. 
partially occluded objects. Occlusion is a pervasive occurrence in ordinary scenes. To perceive complete objects despite occlusion is one of the most basic mandates and successes of visual perception.

The theory applies to subjective figures in the same way as it does to partially occluded objects. A detailed argument for this claim is presented in Kellman and Shipley (in press) and Kellman and Loukides (1987). Figure 10 illustrates equivalent examples of unit formation whose surface appearances differ. In all four displays, a central unit is seen despite the lack of physical specification along parts of its boundary. In each case, three contours have been interpolated between edges with $D_{1} s$ to form a complete unit. The difference in appearance between Figures $10 \mathrm{a}$ and $10 \mathrm{~b}$, that the subjective figure is seen as "modally" complete while the partially occluded figure is seen as "amodally" complete (Michotte, Thines, \& Crabbe, 1964), is due to the central unit's being assigned as in front of the other units in Figure 10b. Differences in appearance derive from differences in available depth information, not from differences in the interpolation process that produces the central units.

In recent studies (Shipley \& Kellman, 1989), we have found near perfect predictability between the coherence ratings of spatially separated visible parts in occlusion dis-

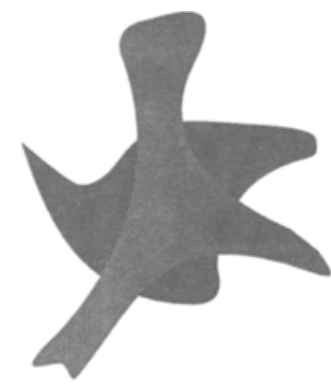

(a)

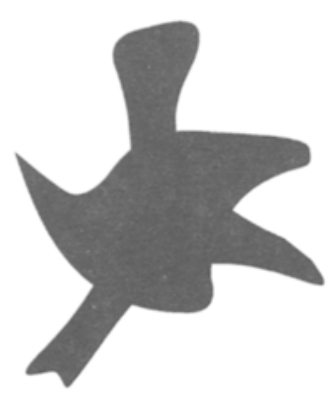

(c)

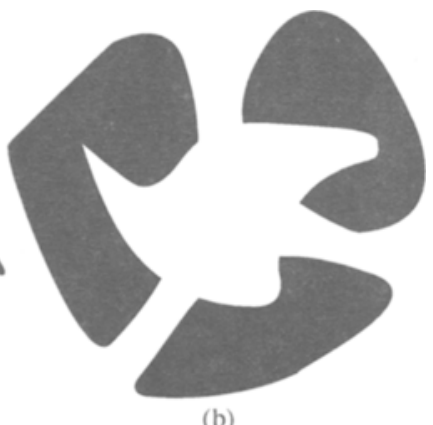

(b)

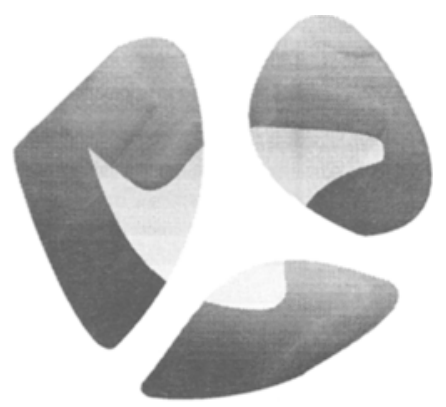

(d)
Figure 10. Equivalent unit formation cases with differing appearances. For the central figure in each case, the same edges are specified by brightness differences, and in each case, the same edges are interpolated through areas where they are not specified. (a) A partly occluded figure. (b) A subjective figure. (c) A spontaneously splitting figure. (d) A transparent subjective figure.

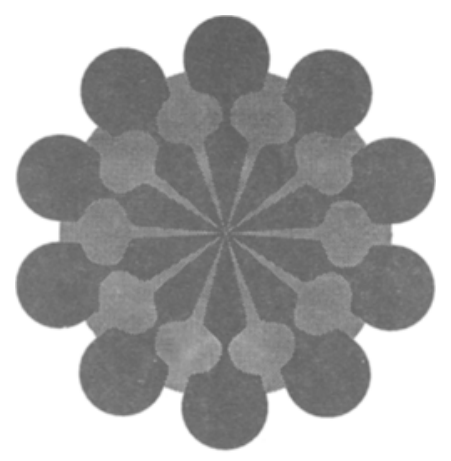

a)

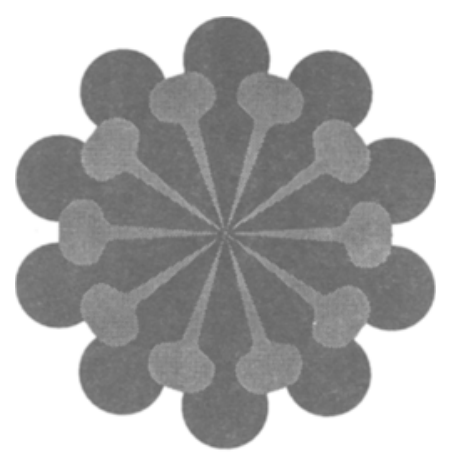

b)

Figure 11. An example of a partially occluded discontinuity-absent and discontinuity-present display. (a) The gray regions have discontinuities in the first derivative of their edges; as a result, a gray circle is seen behind multiple black figures. (b) The discontinuities on the gray regions have been removed; the gray parts are now seen in front of the black figures.

plays and the clarity ratings of equivalent subjective figures. These results support the notion of a single process responsible for perception of subjective figures and perception of occluded figures. On the basis of the present results, we expect $D_{1} s$ to play a similar role in the perception of partially occluded figures. Experiments on unit formation in occlusion displays that are formally equivalent to Experiments 1, 2, 3, and 4 should produce equivalent results. Figure 11 gives an example of two partially occluded figure displays that are based on subjective figure displays used in Experiment 4. The percept of a partially occluded gray circle appears to be much stronger in Figure 11a, where the gray parts of the circle have $D_{1} s$, than in Figure $11 \mathrm{~b}$, where the gray areas have no $D_{1} s$.

The fact that the gray areas in Figure $11 \mathrm{~b}$ seem to be above the black figure while the gray areas in Figure 11a appear to be part of a figure under the black figure suggests an interesting relationship between $D_{1} s$ and the depth cue of interposition. As we have suggested elsewhere (Kellman \& Shipley, in press), one unit may appear to be behind a second when the surfaces of two units (at least one with interpolated boundaries) lie in the same visual 
direction. Following the boundaries of each unit in the projection, the unit whose surface quality changes at the boundary intersection is seen as behind the other. Because $D_{1} s$ are necessary for such interpolation, one figure should only be seen to be behind another when the visible parts of the far figure contain $D_{1} s$. We are currently investigating some of the predictions suggested by this claim.

\section{REFERENCES}

Brigner, W. C., Gallagher, M. B. (1974). Subjective contour: Apparent depth or simultaneous brightness contrast? Perceptual \& Motor Skills, 38, 1047-1053.

Buffart, H., Leeuwenberg, E., \& Restle, F. (1981). Coding theory of visual pattern completion. Journal of Experimental Psychology: Human Perception \& Performance, 7, 241-275.

COREN, S. (1972). Subjective contours and apparent depth. Psychological Review, 79, 359-367.

DAY, R. H. (1987). Cues for edge and the origin of illusory contours: An alternative approach. In S. Petry \& G. E. Meyer (Eds.), The perception of illusory contours (pp. 53-61). New York: Springer-Verlag.

DAY, R. H., \& KASPERCZYK, R. T. (1983). Amodal completion as a basis for illusory contours. Perception \& Psychophysics, 33, 355-364.

HALPERN, D. F. (1981). The determinants of illusory-contour perception. Perception, 10, 199-213.

Kanizsa, G. (1979). Organization in vision. New York: Praeger.

Kellman, P. J., \& Loukides, M. G. (1987). An object perception approach to static and kinetic subjective contours. In S. Petry \& G. E. Meyer (Eds.), The perception of illusory contours (pp. 151-164). New York: Springer-Verlag.

Kellman, P. J., \& Shipley, T. F. (in press). Visual interpolation in object perception: A computational theory. Cognitive Psychology.

Kennedy, J. M. (1978). Illusory contours and the ends of lines. Perception, 7, 605-607.

Michotte, A., Thines, G., \& Crabbe, G. (1964). Les compléments amodaux des structures perceptives. Louvain: Publications Universitaires de Louvain.

PARKs, T. E. (1986). Illusory figures, illusory objects, and real objects Psychological Review, 93, 207-215.
Petry, S., Harbeck, A., Conway, J., \& Levey, J. (1983). Stimulus determinants of brightness and distinctness of subjective contours. Perception \& Psychophysics, 34, 169-174.

Petry, S., \& Meyer, G. E. (Eds.) (1987). The perception of illusory contours. New York: Springer-Verlag.

RoCK, I. (1983). The logic of perception. Cambridge, MA: MIT Press.

SamBin, M. (1987). A dynamic model of anomalous figures. In S. Petry \& G. E. Meyer (Eds.), The perception of illusory contours (pp. 131142). New York: Springer-Verlag.

Schumann, F. (1904). Einige Beobachtungen über die Zusammenfassung von Gesichtseindrücken zu Einheiten. Psychologische Studien, 1, 1-32.

SHIPLEY, T. F. (1988). Unit formation in subjective contour perception: Evidence for a discontinuity theory. Unpublished doctoral dissertation, University of Pennsylvania.

Shipley, T. F., Kellman, P. J. (1988, November). Discontinuity theory and the perception of illusory figures. Paper presented at the meeting of the Psychonomic Society, Chicago.

Shipley, T. F., Kellman, P. J. (1989, November). Visual interpolation in the third and fourth dimensions. Paper presented at the meeting of the Psychonomic Society, Atlanta.

van Tuis, H., \& Leeuwengerg, E. (1982). Peripheral and central determinants of subjective contour strength. In H. G. Geissler, P. Petzoldt, H. F. J. M. Buffart, \& Y. M. Zabrodin (Eds.), Psychological judgment and the process of perception (pp. 114-129). Amsterdam: North-Holland.

Wallach, H., Slaughter, V. (1988). The role of memory in perceiving subjective contours. Perception \& Psychophysics, 43, 101-106.

\section{NOTES}

1. It is likely that some areas of extremely sharp curvature, which are not mathematically discontinuous, may be treated by the visual system as discontinuities; this possibility will be examined later in connection with interpretation of some experimental results.

2 . The contrast in the figures shown to subjects has been reversed for purposes of reproduction.

3. We wish to thank Ed Pugh for first suggesting this possibility.

(Manuscript received August 16, 1989; revision accepted for publication April 24, 1990.)

\title{
Notices and Announcements
}

\author{
20th Annual Meeting of the Society for Computers in Psychology \\ New Orleans, Louisiana \\ November 15, 1990
}

The 20th Annual Meeting of the Society for Computers in Psychology will be held at the Hyatt Regency Hotel in New Orleans on November 15, 1990. The meeting will include presentations, discussions, and times to preview software and hardware. All areas of psychology are featured, including research, education, clinical practice, and industrial applications.

For further information, contact Sarah Ransdell, Department of Psychology, University of Maine, Orono, ME 04469 (BITNET address RANSDELL@MAINE) or C. Michael Levy, Department of Psychology, University of Florida, Gainesville, FL 32611 (BITNET address MLEVY@UFFSC). 